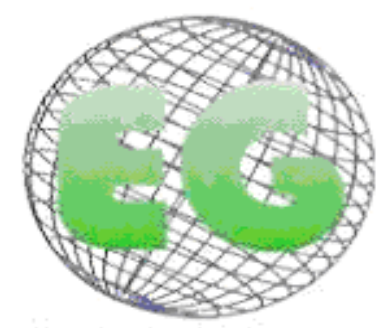

ISSN 1695.6141 $N^{\circ} 26$

\title{
Una guía clara, aplicable, recomendable y que se cumple
}

A clear, applicable and recommendable guide with high acceptance

\section{*Rodríguez García, MJ., " ${ }^{* *}$ el Castillo Arévalo, F., ${ }^{* * *}$ Arenas Lobeto, A., ${ }^{* * *}$ Fernández Fernández, I. ${ }^{* * *}$ Martinez González, C. ***Pérez Miras, AR.}

\author{
*Enfermera. Centro de Salud de Infiesto. E-mail: amahuaca83@gmail.com **Enfermera. Centro de Salud de \\ Contrueces. ***Escuela Universitaria de Enfermería SESPA. Gijón.
}

Palabras clave: guía; cuidadores; enfermería de Atención Primaria; promoción de la salud; educación en salud.

Keywords: guide; caregivers; primary care nursing; health promotion; health education.

\section{RESUMEN}

Objetivos: Conocer la utilización de la guía "Atención a las personas cuidadoras" por las enfermeras que han realizado intervenciones grupales con cuidadoras y averiguar su opinión sobre:

- la idoneidad y estructura de las unidades didácticas

- la necesidad de modificación del resto de contenidos: organización del Servicio, registro, encuesta inicial, cuestionario de calidad de vida, de evaluación del taller.

- los temas no tratados que sería recomendable introducir.

Metodología: Estudio descriptivo transversal mediante una encuesta aplicada a las enfermeras de Atención Primaria de Asturias que realizaron Talleres grupales con cuidadores familiares durante el periodo enero 2009junio 2010.

Resultados: El porcentaje de respuesta al cuestionario fue del 81\%. La guía fue empleada para la preparación de la intervención por el $95 \%$ de las profesionales. El 96\% de las encuestadas manifiestan que los contenidos de la guía responden a las necesidades de los cuidadores. Mayoritaria es también la respuesta de quienes la consideran comprensible y fácil de manejar, el $92 \%$. Destacaron la falta de formación como dificultad para impartir los contenidos de la guía el $77 \%$. El $91 \%$ de las encuestadas recomendaría esta guía para el desarrollo de una intervención grupal con personas cuidadoras.

Conclusiones: Es bastante elevada la adherencia de las enfermeras a esta guía que orienta la intervención psicoeducativa grupal con cuidadores de personas dependientes. Se requiere llevar a cabo estrategias útiles por parte de la Administración Sanitaria con el fin de resolver las barreras identificadas que dificultan su mejor uso y aplicación, entre ellas, mejorar la formación de las enfermeras en habilidades de comunicación y de inteligencia emocional. Es necesario realizar una revisión y actualización de los contenidos de la guía para que sea una herramienta que ayude y mejore la calidad de la práctica de este Servicio. 


\title{
ABSTRACT
}

Objectives. To assess the use of the guide "Atención a las personas cuidadoras" ("Care for caregivers") by nurses who had performed group interventions with caregivers and to obtain their opinion on:

- The suitability and structure of the didactic units

- The need to modify the rest of the contents: Department organization, registration, initial survey, quality of life questionnaire, workshop review questionnaire

- Topics which are not dealt with but which should be included

\begin{abstract}
Methods. Transversal descriptive study. A survey of primary care nurses in Asturias who had conducted group
\end{abstract} workshops with family caregivers between January 2009 and June 2010 was carried out.

Results. The response percentage to the survey was $81 \%$. The guide was used to prepare interventions by $95 \%$ of the professionals. $96 \%$ of the survey group declared that the contents of the guide address the needs of the caregivers. Most nurses, $92 \%$, also consider the guide to be easy to understand and to use. $77 \%$ of them identified a lack of training as a difficulty when trying to teach the contents of the guide. Finally, $91 \%$ of the survey group would recommend using this guide when developing a group intervention with caregivers.

Conclusions. Nurse adherence to this guide, which helps direct the psychoeducational group intervention for caregivers of dependent persons, was remarkably high. The Healthcare Administration should implement useful strategies in order to overcome the identified barriers which hamper a better use and application of the guide. Its contents must be reviewed and updated so that the guide may become a helpful tool capable of improving the quality of care in this Department.

\section{INTRODUCCIÓN}

En el año 2005, la Subdirección de Atención Primaria del Servicio de Salud de Asturias incluyó en la Cartera de Servicios de Atención Primaria la atención grupal a las personas cuidadoras de familiares en situación de dependencia. El nuevo Servicio se denominó: "Educación para la salud a grupos y actividades comunitarias: personas cuidadoras". La atención a este grupo de personas fue una de las prioridades acordadas entre la Consejería de Salud y Servicios Sanitarios, el SESPA (Servicio de Salud del Principado de Asturias ) y el Instituto Asturiano de la Mujer para promocionar la salud y calidad de vida de dicho colectivo integrado en su mayor parte por mujeres.

Es abundante la literatura que pone de manifiesto la sobrecarga física y emocional asociada al desempeño del rol del cuidador, así como las repercusiones familiares, laborales, económicas, en el ocio y tiempo libre que soportan estas personas. ${ }^{1-4}$

Así mismo, en las revisiones recientes sobre los resultados de los programas con cuidadores, se señala como una de las limitaciones la escasa información sobre sus características y los procedimientos empleados. Para subsanar ese tipo de carencias, se recomienda ${ }^{5}$, entre otras cosas, que los contenidos de la intervención sean descritos detalladamente, para que puedan ser reproducidos por otros profesionales y generalizar sus conclusiones a partir de estudios metodológicamente rigurosos.

Como material de apoyo a las profesionales para la realización de dicha actividad, la Coordinación de Enfermería del SESPA propuso la elaboración de una guía de actuación ${ }^{6}$ Para tal fin se constituyó un grupo de trabajo que examinó las guías existentes en ese momento ${ }^{7,8}$, algunos textos relacionados con el impacto del cuidar en la vida de los cuidadores y estrategias de intervención llevadas a cabo por el sistema formal con dichas personas. Para acceder a estas fuentes de información, se realizó una búsqueda bibliográfica en las bases de datos de los Servicios de Salud de las Comunidades 
Autónomas, en Medline, en la Biblioteca Cochrane, en la página web del IMSERSO y en la página web de la Sociedad Española de Geriatría y Gerontología.

Los textos consultados fueron de gran ayuda, aunque insuficientes para la finalidad pretendida, que consistía en facilitar a las enfermeras un manual que recogiera las actuaciones que la evidencia disponible había mostrado ser más efectivas ${ }^{9-12}$ en la reducción del malestar de los cuidadores familiares.

La guía elaborada que lleva por título "Atención a las personas cuidadoras", consta de nueve unidades didácticas (Tabla I), orientaciones sobre cómo organizar el Servicio en el centro de salud, una encuesta inicial para los cuidadores, un cuestionario de calidad de vida del cuidador (WHQOL-BREF) para evaluar el impacto de la intervención a pasar en tres momentos diferentes, uno antes de su inicio, otro al finalizar y otro a los 9 meses, y un cuestionario de evaluación del taller (Tabla II).

Tabla I
Unidades Didácticas
\begin{tabular}{|l|l|}
\hline 1 & El sistema informal de cuidados. ¿Quién cuida hoy y porqué? \\
\hline 2 & Los cuidados que presta la cuidadora. ¿En qué consiste cuidar? \\
\hline 3 & $\begin{array}{l}\text { Dime lo que piensas y te diré como te sientes. Modelo de Situación- } \\
\text { Pensamientos-Sentimientos }\end{array}$ \\
\hline 4 & Emociones y Sentimientos \\
\hline 5 & La Cormunicación interpersonal \\
\hline 6 & Final de vida y pérdidas \\
\hline 7 & El estrés, una respuesta humana \\
\hline 8 & Cuidados de la persona dependiente \\
\hline 9 & Cuidar la propia salud. Recursos sociales \\
\hline
\end{tabular}




\begin{tabular}{|l|} 
Tabla II \\
Componentes de la Guia de Atención a las Personas Cuidadoras \\
\hline Introducción \\
\hline Unidades Didáctic as \\
\hline Organización funcional del Servicio \\
\hline Hoja de derivación para el Taller grupal de apoyo a las personas cuidadoras \\
\hline Encuesta inicial a las personas cuidadoras \\
\hline Cuestionario de calidad de vida WHOQOL-BREF \\
\hline Evaluación del taller \\
\hline
\end{tabular}

Cada unidad didáctica está compuesta por varios apartados: introducción, objetivos, desarrollo metodológico, fichas del formador, fichas de trabajo, fichas-resumen y bibliografía. Las fichas del formador orientan al profesional sobre los objetivos a desarrollar en la sesión, conceptos, actitudes y procedimientos a trabajar con el grupo. Las fichas de trabajo recogen la propuesta de actividades a llevar a cabo por los cuidadores.

La evaluación de este tipo de documentos es una práctica necesaria ${ }^{13}$ si se quiere mejorar su calidad y evitar que sus carencias se perpetúen en el tiempo. Por este motivo, después de 5 años desde su publicación y difusión, se ha realizado este estudio cuyos objetivos son conocer:

a) la utilización de la guía por las enfermeras que han realizado intervenciones grupales con cuidadoras y

b) su opinión sobre:

- la idoneidad y estructura de las unidades didácticas

- la necesidad de modificación del resto de contenidos: organización del Servicio, registro, encuesta inicial, cuestionario de calidad de vida, de evaluación del taller.

- los temas no tratados que sería recomendable introducir.

\section{MATERIAL Y MÉTODO}

Estudio descriptivo transversal mediante una encuesta aplicada en diciembre de 2010 en la Comunidad Autónoma de Asturias.

La población diana la forman las enfermeras de Atención Primaria que realizaron Talleres grupales con cuidadores familiares durante el periodo enero 2009- junio 2010. 
Las Direcciones de Enfermería de cada Área Sanitaria facilitaron la relación de las 47 profesionales que cumplían el criterio de inclusión.

Se contactó telefónicamente con cada una de ellas para explicarles el proyecto e invitarles a participar. Posteriormente, se les envió por correo postal o por correo electrónico la encuesta y una carta de presentación.

Se realizó una segunda y tercera llamada telefónica recordatoria a las 3 y 5 semanas a las personas que no habían contestado. Se recibieron 38 encuestas de las cuales se excluyeron 3 que no habían utilizado la guía. Los sujetos de estudio son la población de 35 personas que cumplen los criterios de inclusión.

El grupo investigador elaboró una encuesta que fue pilotada por 5 enfermeras que habían utilizado la guía para asegurar la relevancia y comprensión de las preguntas. Con los comentarios y sugerencias aportados, se redactó el cuestionario definitivo formado por 23 preguntas, 21 de ellas cerradas, que recogen: aspectos generales de la Guía, como facilidad de manejo, utilidad, comprensión, claridad y acuerdo con los contenidos; en una segunda parte las variables relacionadas con las Unidades Didácticas, estructura y contenidos; en la tercera parte se interroga sobre el registro de este servicio en la historia clínica, el cuestionario inicial y de calidad de vida que se aplica a los cuidadores y el de evaluación del taller. Una de las preguntas abiertas indaga sobre los temas que deberían ser incluidos y la otra ofrece a las encuestadas la oportunidad de realizar comentarios adicionales.

El análisis descriptivo de los datos se realizó calculando las medidas de tendencia central y dispersión (media y desviación estándar) para las variables cuantitativas y frecuencias absolutas y proporciones e intervalos de confianza del $95 \%$ para las variables cualitativas.

\section{RESULTADOS}

De las 47 encuestas enviadas, respondieron 38 enfermeras lo que eleva el porcentaje de respuesta al $81 \%$. La guía fue empleada para la preparación de la intervención por el $95 \%$ de las profesionales.

De los 69 talleres impartidos durante el periodo del estudio, el 28\% fueron realizados por una enfermera como único profesional participante, alcanzando el $72 \%$ los que contaron además con la colaboración de otro profesional que fue mayoritariamente una trabajadora social (64\%).

El $96 \%$ de las encuestadas manifiestan que los contenidos de la guía responden a las necesidades de los cuidadores. Mayoritaria es también la respuesta de quienes la consideran comprensible y fácil de manejar, el $92 \%$. la percibe útil, y suficiente como material de apoyo el 55\%, pero están en desacuerdo con esa afirmación el 34\%.

Destacaron la falta de formación como dificultad para impartir los contenidos de la guía el $77 \%$ (Gráfico 1). Entre quienes dicen discrepar con algunos de sus contenidos y por tal motivo no abordarlos en el taller, se hallan el $37 \%$ de las enfermeras. 


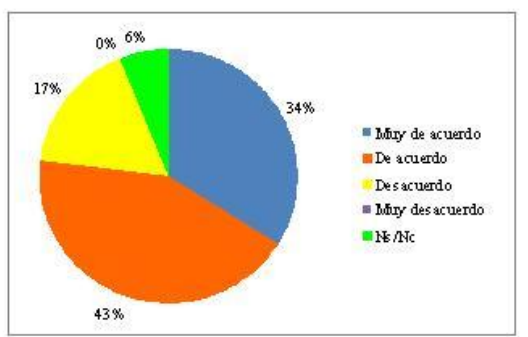

Gráfico 1. La falta de formación dificulta impartir los contenidos de la guía

El 80\% manifestó tratar las 9 unidades didácticas, un $6 \%$ no programaron nunca o solo a veces las unidades 3 (Pensamientos disfuncionales), 4 (Emociones y sentimientos), y 5 (Comunicación interpersonal), ascendiendo ese porcentaje al 15\% para la unidad 6 (Final de la vida y las pérdidas). Respecto a la necesidad de efectuar cambios en los contenidos de las unidades, el $55 \%$ optó por la unidad 3 (Pensamientos disfuncionales), el 54\% por la Unidad 8 (Cuidados a la persona dependiente) y el $46 \%$ por la 4 (Emociones y sentimientos), 6 (Final de vida y pérdidas) y 7 (El estrés, una respuesta humana). (Gráfico 2).

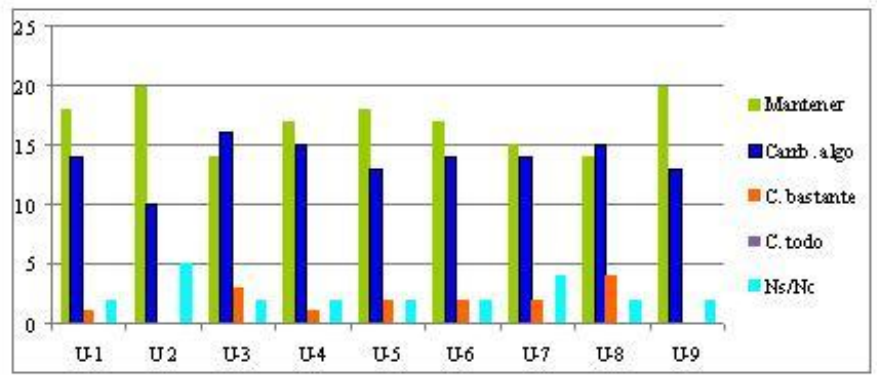

Gráfico 2. Opiniones sobre los contenidos de las unidades didácticas 
Interrogadas las participantes sobre si las fichas del formador les proporcionaban la información necesaria para impartir la unidad, una amplia mayoría dijo estar de acuerdo con esa afirmación. El rango oscila entre el $69 \%$ de la unidad 5 (Comunicación interpersonal) y el $95 \%$ de las unidades 1 (Sistema informal de cuidados) y 9 (Cuidar la propia salud. Recursos sociales).

Las fichas de trabajo que acompañan a cada unidad didáctica recogen la propuesta de actividades a llevar a cabo por los cuidadores. A los investigadores les interesa conocer si tal como están diseñadas facilitan el desarrollo de las sesiones. También en este apartado una considerable mayoría respondió afirmativamente. Los porcentajes más bajos de acuerdo fueron para las unidades 6 (Final de la vida y pérdidas) con un 74\% y para la unidad 8 (Cuidados a la persona dependiente) con un $76 \%$.

Algo más de la mitad, 54\%, son partidarias de realizar cambios en el registro en la historia clínica de este servicio tal y como se efectúa ahora, frente al 31\% que lo mantendría como está. En cuanto a la encuesta inicial que se administra a los cuidadores para conocer sus necesidades y circunstancias en las que prestan el cuidado, el $80 \%$ manifestó realizarla siempre, cifra que se eleva a $97 \%$ si se incluye la opción "casi siempre". Aún así, el $60 \%$ considera que debe ser objeto de modificaciones.

El 37\% lo aplicó "siempre" antes del comienzo, descendiendo al 9\% quienes lo llevaron a cabo "siempre" al finalizar las sesiones y a los 9 meses de la intervención. Para el $63 \%$ no debe mantenerse como instrumento de evaluación y una de cada 5 "no sabe/no contesta".

Cuenta con bastante aceptación, $69 \%$ el modelo de derivación de los cuidadores a la enfermera coordinadora del taller, a utilizar por los profesionales del centro de salud.

Optaron por mantener el cuestionario de evaluación del taller el $51 \%$ si bien para el $43 \%$ se precisaría realizar modificaciones en el mismo.

El 91\% de las encuestadas recomendaría esta guía para el desarrollo de una intervención grupal con personas cuidadoras.

Entre los temas que incluirían, mencionaron: nutrición, higiene de la espalda del cuidador, autoestima, asertividad, inteligencia emocional, y cuidados a la persona dependiente.

\section{DISCUSIÓN}

Cabe destacar que el alto porcentaje de respuesta al cuestionario, 81\%, nos permite tener una imagen muy fidedigna de lo que opinan las enfermeras que realizan intervenciones grupales con cuidadores sobre la guía propuesta por el SESPA como recurso de ayuda en la implantación de este servicio en A.P.

La Guía tiene bastante éxito como lo prueba que una amplísima mayoría, 95\%, la utiliza en la programación de los talleres, cifra notablemente superior a la alcanzada por otras guías ${ }^{14,15}$. Los datos permiten afirmar que el texto, en líneas generales, es muy bien valorado y cuenta con un amplio respaldo entre sus principales destinatarias.

Mayoritariamente, 92\%, consideran que responde satisfactoriamente a algunas de las características de lo que en la bibliografía se considera "una buena guía" ${ }^{16}$. El manual es comprensible, manejable, las unidades didácticas recogen los problemas y necesidades de los cuidadores familiares, y la recomiendan como material de apoyo (Tabla I). 
Que sea considerada útil y suficiente por el $55 \%$ de las enfermeras puede ser debido al desacierto en la formulación de la pregunta al interrogar en la misma sobre dos conceptos que tienen diferente significado y/o a que echan en falta algunos temas que deberían estar incluidos y por tal motivo les resulta insuficiente.

En la bibliografía consultada en las bases de datos MEDLINE, IBECS, IME, CUIDEN, CUIDEN plus, PUBMED, no se han hallado estudios sobre la evaluación del contenido de las guías que dan soporte a las intervenciones grupales psicoeducativas con cuidadores familiares, por lo que no se han podido contrastar algunos de los resultados obtenidos.

Se han identificado distintos factores como causa de la falta de adherencia de los profesionales a las recomendaciones de las guías ${ }^{17}$. Uno de ellos es la falta de conocimientos y habilidades como motivos que impiden la aplicación de sus contenidos ${ }^{18}$. En este estudio, la barrera relacionada con la percepción de falta de competencia es manifestada por el $77 \%$. Otra de las dificultades descritas en la bibliografía ${ }^{19}$ es la que tiene que ver con las actitudes y opiniones de los profesionales. Esta podría ser la explicación de que por discrepancias con algunos de los contenidos de la guía, el 37\% de las encuestadas decida no tratarlos en los talleres.

Una de las limitaciones del estudio es no indagar sobre las barreras y facilitadores en el seguimiento de las recomendaciones que están relacionados con el contexto social y organizativo.

Por tratarse de una guía de ayuda a la implantación de una intervención psicoeducativa, cuatro de las nueve unidades están relacionadas con las necesidades psicológicas y emocionales de los cuidadores (Tabla II). El documento incluye entre otros contenidos, estrategias para el manejo de emociones como la tristeza y la culpa, la identificación de los pensamientos disfuncionales y la reestructuración cognitiva, el entrenamiento en la comunicación asertiva y el aprendizaje de técnicas de relajación. Son en estos temas en los que se concentra la mayor proporción de solicitud de modificaciones. Le sigue la unidad que trata de los cuidados a la persona dependiente, y a continuación la del final de la vida y las pérdidas.

Una explicación plausible de estos datos es que las enfermeras priorizan aquellos temas relacionados con los cuidados a la persona dependiente, en los que se encuentran más cualificadas, sobre el entrenamiento en habilidades instrumentales y emocionales del cuidador para lo que requieren más aprendizaje. Otorgan mayor preferencia a "cómo cuidar" que a "cómo cuidarse" el cuidador.

También y en mayor medida se ve la necesidad de reformar el registro del servicio en la historia clínica del cuidador, la encuesta inicial, el cuestionario que trata de medir el impacto de la intervención y el de evaluación final del taller.

En relación con la incorporación de nuevos temas descritos anteriormente, algunos autores ${ }^{11}$ señalan que el exceso de contenidos dificulta el aprendizaje efectivo de habilidades y su consolidación. Teniendo en cuenta la escasa disponibilidad de tiempo libre de los cuidadores, se desaconsejan ${ }^{20}$ intervenciones que requieran más de 8 ó 10 sesiones, pues una duración superior podría disuadir su participación. Es preciso tener presentes este tipo de consideraciones para alcanzar resultados más satisfactorios.

Como línea futura de investigación se recomienda explorar qué tipo de innovaciones son pertinentes efectuar para mejorar la calidad del documento. 
El estudio podría presentar un sesgo en la información facilitada por las encuestadas al no haberse garantizado el anonimato, ya que los cuestionarios fueron remitidos por e-mail y devueltos por esta misma vía una vez cumplimentados. Las respuestas podrían haber sido distorsionadas en sentido favorable debido a la influencia de "la deseabilidad social".

\section{CONCLUSIONES}

Los datos del estudio permiten concluir: a) que es elevada la adherencia de las enfermeras a la guía que sirve de ayuda para llevar a cabo una intervención psicoeducativa grupal con cuidadores de personas dependientes; b) que se requieren llevar a cabo estrategias útiles por parte de la Administración Sanitaria con el fin de resolver las barreras identificadas que dificultan su mejor uso y aplicación entre las que se encuentra la mejora de la formación de las enfermeras en habilidades de comunicación y de inteligencia emocional; c) que es necesario realizar una revisión y actualización de los contenidos de la guía para que sea una herramienta que ayude y mejore la calidad de la práctica de este Servicio.

\section{BIBLIOGRAFÍA}

1. Badia X, Lara N, Roset M. Calidad de vida, tiempo de dedicación y carga percibida por el cuidador principal informal del enfermo de Alzheimer. Aten Primaria. 2004; 34:170-177.

2. Crespo M, López J. El estrés en cuidadores de mayores dependientes. Cuidarse para cuidar. 2007. Ed. Pirámide. Madrid.3. Pinquart M, Sörensen S. Associations of caregiver stressors and uplifts with subjective well-being and depressive mood: a meta-analytic comparison. Aging Ment Health. 2004 Sep;8(5):438-49.

4. Saiz Ladera G M, Bordallo Huidobro J R, García Pascual J N. El cuidador como paciente FMC. 2008; 15(7):418-26

5. Izal Fernández de Trocóniz, M, Montorio Cerratol , Márquez González M, Losada Baltar A. Estudio e intervención sobre el malestar psicológico de los cuidadores de personas con demencia. El papel de los pensamientos disfuncionales.

Instituto de Mayores y Servicios Sociales (IMSERSO). 2006

6. Guía de Atención a las personas cuidadoras. Servicio de Salud del Principado de Asturias 2006.

7. Transiciones y etapas vitales vulnerables. Experiencias de educación para la salud en Atención Primaria 1987-1997. Servicio Navarro de Salud. 1998. Navarra.

8. Taller de formación y educación sanitaria para personas cuidadoras. Dirección General de Programas Asistenciales. Servicio de Atención Primaria, Planificación y Evaluación. Servicio Canario de Salud. 2003

9. Sörensen S, Pinquart M, Duberstein P. How effective are interventions with caregivers? An updated meta-analysis. Gerontologist. 2002; 42(3):356-72.

10. Losada-Baltar A, Izal-Fernández de Trocóniz M, Montorio-Cerrato I, Márquez González M., Pérez Rojo G. Eficacia diferencial de dos intervenciones psicoeducativas para cuidadores de familiares con demencia. Rev. Neurología. 2004; 38(8):701-708

11. Gallagher-Thompson D, Lovett S, Rose J, McKibbin C, Coon D, Futterman A., Thompson LW. Impact of Psychoeducational Interventions on Distressed Family Caregivers. Journal of Clinical Geropsychology. 2000; 6: 91-110.

12. Losada-Baltar A., Montorio Cerrato I. Pasado, presente y futuro de las intervenciones psicoeducativas para cuidadores familiares de personas mayores dependientes. Rev. Esp. Geriat. Gerontol. 2005; 4 (Supl 3): 30-9.

13. Alonso P, Bonfill X. Guías de práctica clínica (I): elaboración, implantación y evaluación. Radiología. 2006; 49(1):19-22. 
14. Ruiz-García A, Gordillo-López F J, Arranz-Martínez E, Morales-Cobos L E, FerrariFerrando C. Valoración de la implantación en Atención Primaria de la guía de práctica clínica ERGE. Semergen. 2008; 34 (9):439-43.

15. Fernández Sánchez A, Sánchez-Carracedo D, Navarro-Rubio M, Pinto-Meza A, Moreno-Küstner B. Opiniones de médicos de atención primaria, psiquiatras y psicólogos acerca de las guías de práctica clínica para la depresión. Un estudio cualitativo exploratorio. Aten Primaria.2010; 42:552-8.

16. Castiñeira Pérez C, Medrano Ortega F.J. ¿Cómo leer una GPC?

http://www.fisterra.com/guias2/FMC/leer.asp (Consulta realizada el 21/ 08/2011)

17. Grupo de trabajo sobre implementación de GPC. Implementación de guías de práctica clínica en el sistema nacional de salud. Manual metodológico. Ministerio de Ciencia e Innovación. $2009 . \quad$ Disponible en http://www.guiasalud.es/emanuales/implementacion/documentos/Manual Implementacion.pd f. (Consulta realizada el 21/8/2011).

18. Grol R, Dalhuijsen J. Attributes of clinical guidelines that influence use of guidelines in general practice: observational study. BMJ. 1998; 317: 858-861.

19. Martín Zurro A, Cano Pérez J P. Conceptos, organización y práctica clínica. Vol. I. Ed Elsevier España. 2005.

20. Knight B G, Lutzky S M., Macofsky-Urban F. A Meta-analytic Review of Interventions for Caregiver Distress: Recommendations for Future Research. The Gerontologist. 1993; 33(2), 240-248. 